\title{
Orientation Dependent Field Evaporation Behavior in Multilayer Thin Films
}

\author{
J.G. Brons, ${ }^{*}$ A.A. Herzing, ${ }^{* *}$ I.M. Anderson, ${ }^{* *}$ and G.B. Thompson* \\ *Department of Metallurgical \& Materials Engineering, University of Alabama, Tuscaloosa, AL \\ 35487 \\ **Surface \& Microanalysis Science Division, NIST, Gaithersburg, MD 20899
}

Atom probe reconstruction algorithms implicitly assume a constant evaporation field across the surface of the specimen. However, in reality, chemical inhomogeneity modulates the evaporation field, and thereby introduces reconstruction artifacts and degrades the spatial resolution of the atom probe tomography (APT) technique. Quantifying and rectifying the effect of interfacial compositional variations in atom probe data is critical, especially when applied towards understanding elemental intermixing in nanolaminate devices such as giant magnetoresistance multilayers [1]. Multilayer thin films provide a model specimen geometry to measure and quantify these artifacts since they exhibit well defined interfaces with a much greater interfacial-area-to-volume ratio than typical materials.

Two series of alternating pure element multilayers, $\mathrm{Fe} / \mathrm{Ni}$ and $\mathrm{Ti} / \mathrm{Nb}$, have been sputterdeposited onto n-doped [001]-oriented Si substrates. Bilayer repeat units of $\approx 4 \mathrm{~nm}$ featured individual layers of equal $(\approx 2 \mathrm{~nm})$ thickness; a representative STEM-HAADF micrograph of the Fe/Ni specimen is shown in Fig. 1a. APT specimens were prepared from the multilayers by focus ion beam (FIB) milling with an annular raster geometry to achieve the required needleshaped geometry. Specimens of each series of multilayers were prepared in two orientations, with the film interfaces oriented both parallel and perpendicular to the specimen apex, in order to compare field evaporation behavior at these limiting geometries.

Electron energy-loss spectroscopy (EELS) data were acquired from a cross-sectioned specimen of the Fe/Ni multilayer using scanning transmission electron microscopy (STEM) with a spherical aberration-corrected probe $\left(\mathrm{d}_{\mathrm{p}} \approx 100 \mathrm{pm}, \mathrm{i}_{\mathrm{p}} \approx 100 \mathrm{pA}\right)$. Compositional profiles were collected via a STEM-EELS line-scan perpendicular to the interfacial direction, as shown in Fig. 1b. APT analysis was performed to compare field evaporation occurring perpendicularly or parallel to the layer interfaces. Proximity histograms from these reconstructed atom maps are shown in Figs. 1c and 1d, respectively. The compositional profiles from all three analyses show that the multilayer films exhibited intermixing between $\mathrm{Fe}$ and $\mathrm{Ni}$ within the layers.

The compositional gradient ratio of $\mathrm{Fe}$ deposited on $\mathrm{Ni}$ : $\mathrm{Ni}$ deposited on Fe taken from Figs. $1 \mathrm{~b}$ to $1 \mathrm{~d}$ is tabulated in Table 1 . A similar APT-based ratio of Ti deposited on $\mathrm{Nb}: \mathrm{Nb}$ deposited on Ti (profiles not shown) is also tabulated in Table 1. The atom probe ratios for $\mathrm{Fe} / \mathrm{Ni}$ were found to be near unity, which is in reasonably close agreement with the EELS result. Recently, an EELS-based study [2] of $\mathrm{Ti} / \mathrm{Nb}$ multilayers processed under similar conditions and bilayer spacings has reported a profile of the gradient composition ratio near unity too. In contrast, the results of our APT study (Table 1) indicate that a significant deviation from this predicted value is present, and that the difference is of opposite sign depending upon the interfacial orientations.

Less deviation in the reconstruction was observed for the Fe/Ni multilayer, and the results obtained from both orientations more closely agree with the compositional profiles obtained via STEM-EELS. These observations may arise because of the relative difference in field strength between the elements composing the alternating layers. The elemental evaporation fields for $\mathrm{Fe}$ 
$(33 \mathrm{~V} / \mathrm{nm})$ and $\mathrm{Ni}(35 \mathrm{~V} / \mathrm{nm})$ are similar, whereas those for $\mathrm{Ti}(26 \mathrm{~V} / \mathrm{nm})$ and $\mathrm{Nb}(37 \mathrm{~V} / \mathrm{nm})$ differ significantly [3]. Thus, the larger the difference in field strength between elements composing the alternating layers, the greater the discrepancy between the interfacial intermixing measured by EELS and APT. In the parallel orientation, this difference in field strength produces a significant local magnification, manifested as a compression of the lower field strength material (Ti) and an extension in the higher field strength material $(\mathrm{Nb})$. This reconstruction artifact results in an incorrect bilayer spacing in the APT reconstruction.

\section{References:}

1. X.W. Zhou, et al. Acta Materialia 49 (2001) 4005.

2. A. Genc, R. Banerjee, G.B. Thompson, D.M. Maher, A.W. Johnson, \& H.L. Fraser, Ultramicroscopy 109 (2009) 1276.

3. TT Tsong, Surface Science 70 (1978) 211.

4. Research at the University of Alabama is supported by the National Science Foundation (NSF) under grant numbers EPS08-14103 and DMR05-47445.

Table 1. Correlation of Compositional Gradients from Different Characterization Techniques

\begin{tabular}{|c|c|c|c|}
\hline Elemental Multilayers & $\begin{array}{c}\text { EELS Ratio of } \\
\text { Fe on Ni }: \text { Ni on Fe }\end{array}$ & $\begin{array}{c}\text { APT Parallel Ratio } \\
\text { Fe on Ni }: \text { Ni on Fe }\end{array}$ & $\begin{array}{c}\text { APT Perpendicular } \\
\text { Ratio Fe on Ni : Ni on Fe }\end{array}$ \\
\hline $\mathrm{Fe} / \mathrm{Ni}$ & 1.0 & 1.09 & 1.01 \\
\hline & $\begin{array}{c}\text { EELS Ratio of } \\
\text { Fe on Ni }: \text { Ni on Fe }\end{array}$ & $\begin{array}{c}\text { APT Parallel Ratio } \\
\text { Ti on Nb }: \text { Ti on Nb }\end{array}$ & $\begin{array}{c}\text { APT Parallel Ratio } \\
\text { Ti on Nb }: \text { Nb on Ti }\end{array}$ \\
\hline $\mathrm{Ti} / \mathrm{Nb}$ & $\sim 1.0[$ ref. 3] & 1.45 & 0.93 \\
\hline
\end{tabular}
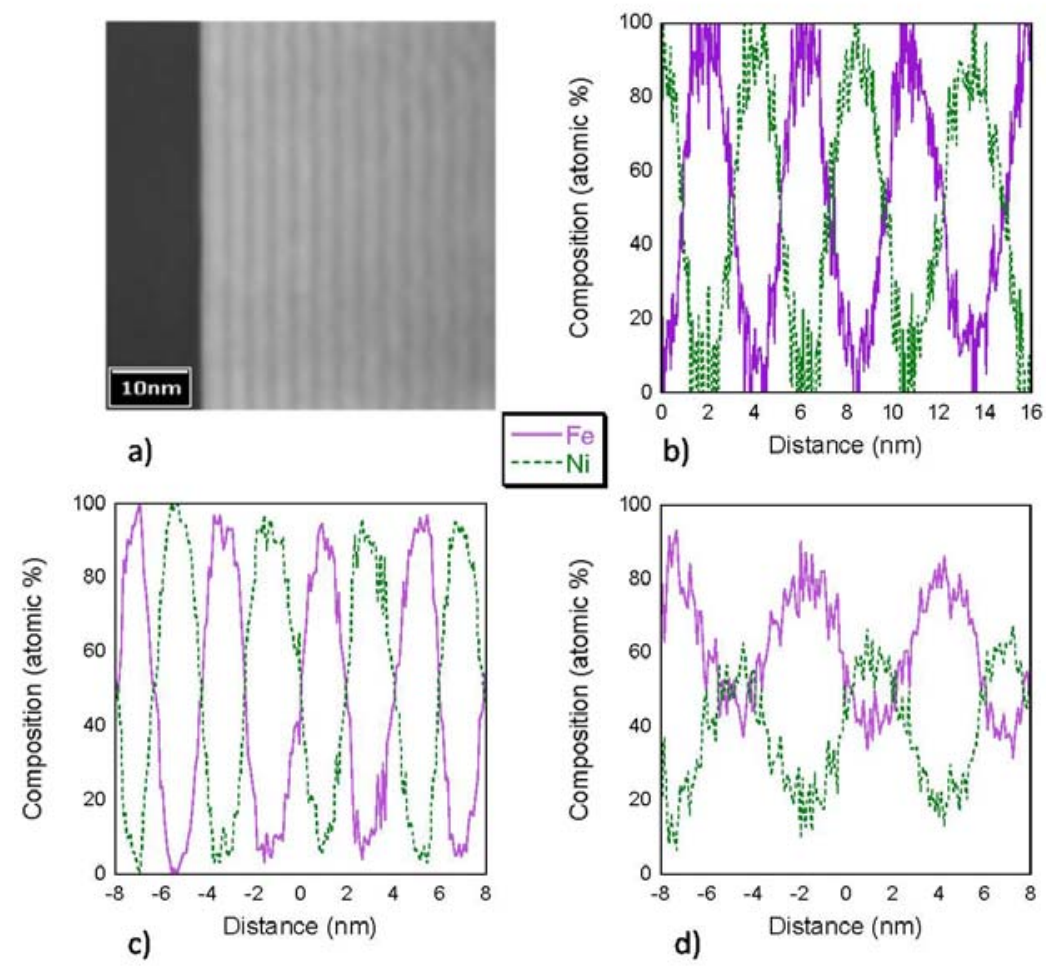

Figure 1. Compositional analysis of an Fe/Ni sputter-deposited multilayer by STEM-EELS and APT: (a) STEM-HAADF micrograph; (b) STEM-EELS compositional profile; (c,d) APT proximity histograms for data acquired in the (c) perpendicular and (d) parallel orientations. 